\title{
Fidelity and Coherence Measures from Interference
}

\author{
Daniel K. L. Oi and Johan Åberg* \\ Centre for Quantum Computation, Department of Applied Mathematics and Theoretical Physics, University of Cambridge, \\ Wilberforce Road, Cambridge CB3 OWA, United Kingdom \\ (Received 27 March 2006; published 29 November 2006)
}

\begin{abstract}
By utilizing single particle interferometry, the fidelity or coherence of a pair of quantum states is identified with their capacity for interference. We consider processes acting on the internal degree of freedom (e.g., spin or polarization) of the interfering particle, preparing it in states $\rho_{A}$ or $\rho_{B}$ in the respective path of the interferometer. The maximal visibility depends on the choice of interferometer, as well as the locality or nonlocality of the preparations, but otherwise depends only on the states $\rho_{A}$ and $\rho_{B}$ and not the individual preparation processes themselves. This allows us to define interferometric measures which probe locality and correlation properties of spatially or temporally separated processes, and can be used to differentiate between processes that cannot be distinguished by direct process tomography using only the internal state of the particle.
\end{abstract}

DOI: 10.1103/PhysRevLett.97.220404

PACS numbers: 03.65.Wj, 03.67.Pp

A defining feature of quantum mechanics is the phenomenon of single particle interference. The ability of a state to display interference, or of a quantum process to preserve this ability, are intuitive notions of coherence and coherent evolution. We elaborate this idea to define interferometric fidelity and coherence measures, generalizing the coherent fidelities between quantum channels introduced in Refs. [1,2]. Interferometry has played an important role in the development of theoretical concepts in quantum mechanics, from which-way experiments [3] to geometric phases [4]. By introducing mixed states and quantum channels into this realm, we obtain a rich structure [5] which we address in this Letter with focus on coherence. Apart from these fundamental aspects, coherence is a prerequisite for quantum information processing. To construct practically useful fault tolerant error correction schemes, it is important to understand the coherence and correlation properties of the processes that act in physical implementations [6]. The measures put forward in this Letter provide means to probe these properties interferometrically.

We briefly review the Mach-Zender interferometer [Fig. 1(a)]. A single particle passes a beam splitter, which causes the particle to traverse two paths in superposition allowing interference at a second beam-splitter. The probabilities to detect the particle at the outputs, $p_{A}=\frac{1}{2}(1+\cos \phi)$ and $p_{B}=1-p_{A}$, depend on a phase shift $\phi$ in one path. The visibility $v=\left[p_{A}\left(\phi_{\max }\right)-\right.$ $\left.p_{A}\left(\phi_{\min }\right)\right] /\left[p_{A}\left(\phi_{\max }\right)+p_{A}\left(\phi_{\min }\right)\right]$ of the interference pattern is unity when the two paths are perfectly coherent, and there is no way, even in principle, to obtain any information about which path the particle "actually" took [3].

We now introduce an internal degree of freedom to the particle (e.g., polarization, spin), described by a Hilbert space $\mathcal{H}_{I}$, and assume that the beam splitters and mirrors do not affect this internal state. A unitary operation $U$ acting on the internal state is placed in one path
[Fig. 1(b)]. If the internal state initially is $|\psi\rangle$, this results in the new interference pattern $p_{A}=\frac{1}{2}[1+v \cos (\phi-\gamma)]$ with visibility $v=|\langle\psi|U| \psi\rangle|$ and phase shift $\gamma=$ $\arg (\langle\psi|U| \psi\rangle)$. The internal state entangles with the path, and path information could be extracted by using the distinguishability between $|\psi\rangle$ and $U|\psi\rangle$ to a degree that corresponds to the reduction of visibility. For a mixed input $\rho$, the interference can be expressed as $F(\rho)=v e^{i \gamma}=$ $\operatorname{Tr}[\rho U]$ [7]. We refer to $F$ as the interference function. The phase shift $\arg F(\rho)$ has been used to define parallel transport of mixed states [8-10], but here we consider the visibility $|F(\rho)|$ of the interference effect.

If we insert into each path a process acting on the internal state of the particle, it seems reasonable to ask
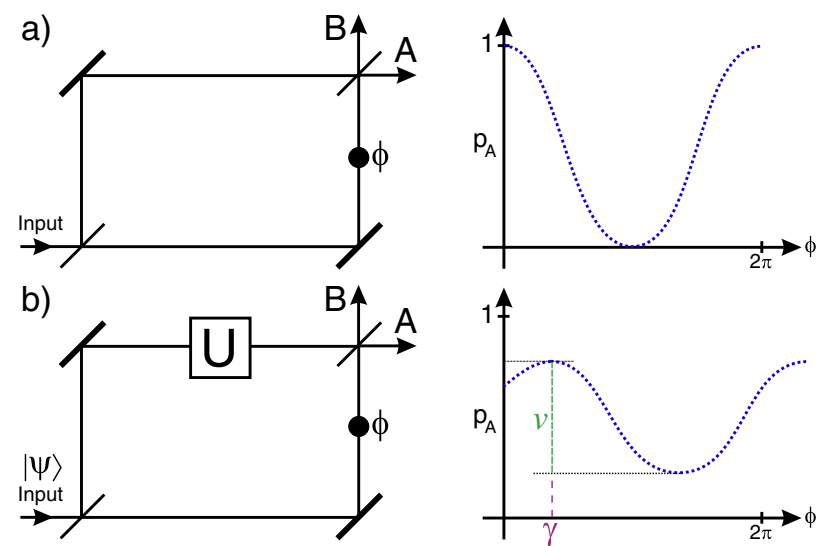

FIG. 1 (color online). (a) Mach-Zender Interferometer. An initial beam splitter places an incident particle into a coherent superposition of traveling along the lower and upper paths. A phase shifter introduces a relative phase shift between the two paths before they recombine on a second beam splitter, and the final direction of the particle is measured. (b) A unitary operation in one path modifies the interference depending on the overlap between the interfering states $|\psi\rangle$ and $U|\psi\rangle$. 
how the interference is modified [Fig. 2(a)]. Suppose these processes can be described by quantum channels, i.e., trace-preserving completely positive maps, $\Lambda_{A}$ and $\Lambda_{B}$ respectively, what would be the corresponding interference function? Surprisingly $[1,2,9,10]$, the interference is not determined solely by $\Lambda_{A}$ and $\Lambda_{B}$ but additional properties of the processes are required in order to uniquely determine $F(\rho)$. More generally, the "marginal" channels $\Lambda_{A}$ and $\Lambda_{B}$ do not uniquely determine the joint operation $\Lambda$ acting on the two paths [5]. Adopting the terminology in Refs. [2,5], a joint channel $\Lambda$ is a gluing of the channels $\Lambda_{A}$ and $\Lambda_{B}$.

In many cases the two processes are independent; e.g., they occur at spacelike separation and do not preshare either classical correlation or entanglement. We call such a total operation a local subspace preserving (LSP) operation [5]. It has been shown [2] that all possible interference functions of LSP gluings of channels $\Lambda_{A}$ and $\Lambda_{B}$ can be written as

$$
F(\rho)=\sum_{k l} b_{l} a_{k}^{*} \operatorname{Tr}\left[A_{k}^{\dagger} B_{l} \rho\right],
$$

where $\|\vec{a}\|_{2},\|\vec{b}\|_{2} \leq 1$, and $\left\{A_{k}\right\}$ and $\left\{B_{l}\right\}$ are arbitrary, but fixed linearly independent Kraus representations [11] of the channels $\Lambda_{A}$ and $\Lambda_{B}$, respectively.

From Eq. (1) one can see that it is possible to define an operator $\tilde{A}_{0}=\sum_{k} a_{k} A_{k}$, and similarly an operator $\tilde{B}_{0}$, such that $F(\rho)=\operatorname{Tr}\left[\tilde{A}_{0}^{\dagger} \tilde{B}_{0} \rho\right]$. In the terminology of Ref. [1] these are the coherence operators of the processes. By a unitary transformation it is always possible to find a Kraus representation with the coherence operator as one of the Kraus operators. The coherence operator then corresponds to the environment being undisturbed by the particle, while the other operators represent cases when the environment experiences a "scattering event" and the coherence of the particle is lost.

Another approach to subspace local gluings is to use Stinespring dilations [12] to represent the channel $\Lambda_{A}(\rho)=\operatorname{Tr}_{E_{A}}\left[\mathbb{U}_{A}\left(\rho \otimes\left|E_{0}^{A}\right\rangle\left\langle E_{0}^{A}\right|\right) \mathbb{U}_{A}^{\dagger}\right]$, where $\left|E_{0}^{A}\right\rangle$ is a state of an environment/ancilla. Using a separate ancilla we can similarly represent $\Lambda_{B}$. It can be shown [2] that all LSP gluings of $\Lambda_{A}$ and $\Lambda_{B}$ can be obtained as $\Lambda(\sigma)=$ $\operatorname{Tr}_{E_{A} E_{B}}\left[\cup \sigma \otimes\left|E_{0}^{A}\right\rangle\left\langle E_{0}^{A}|\otimes| E_{0}^{B}\right\rangle\left\langle E_{0}^{B}\right| \mathbb{U}^{\dagger}\right]$, where $\mathbb{U}=|A\rangle \times$ $\left\langle A\left|\otimes \mathbb{U}_{A} \otimes \mathbb{I}_{B}+\right| B\right\rangle\langle B| \otimes \mathbb{\mathbb { V }}_{A} \otimes \mathbb{U}_{B}$, by varying the

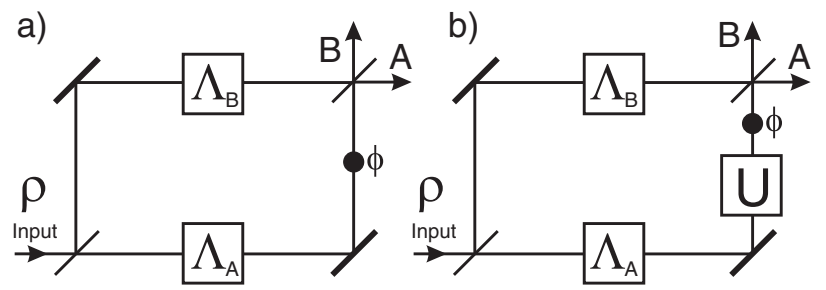

FIG. 2. (a) The internal state of the particle is affected by the channels $\Lambda_{A}$ or $\Lambda_{B}$. The gluing and the interference is not uniquely determined by $\Lambda_{A}$ and $\Lambda_{B}$. (b) A generalized interferometer is obtained by inserting a variable unitary operator. This setup can distinguish all gluings of two given channels.
Stinespring dilations [Fig. 3(a)]. Note that the coherence operators can be written $\tilde{A}_{0}=\left\langle E_{0}^{A}\left|\mathbb{U}_{A}\right| E_{0}^{A}\right\rangle$ and $\tilde{B}_{0}=$ $\left\langle E_{0}^{B}\left|\mathbb{U}_{B}\right| E_{0}^{B}\right\rangle$, which demonstrates that the choice of Stinespring dilations directly determines the LSP gluing and hence the interference. One also sees that the coherence operators indeed correspond to the case when the environment remains unchanged, as mentioned above.

So far we have considered LSP gluings, but we may also consider more general types of gluings. A subspace preserving (SP) channel does not transfer probability weight between the two paths, i.e., the particle does not "jump", but apart from this restriction the process may use any communication or shared classical or quantum correlations [5]. In this case the interference function is similar to Eq. (1), but with $b_{l} a_{k}^{*}$ generalized to a matrix $C_{l k}$ satisfying $C C^{\dagger} \leq I$ [2]. Similarly, as for the LSP gluings, it can be shown [2] that all SP gluings can be reached through various choices of Stinespring dilations of the glued channels, but with the difference that the two paths share a common ancilla [Fig. 3(b)].

The ordinary interferometer has only a limited capacity to determine gluings. By inserting a variable unitary operator in one arm [Fig. 2(b)] we create a generalized interferometer whose generalized interference function $G(\rho, U)$ can distinguish between all SP (and LSP) gluings of two given channels [2],

$$
G(\rho, U)=\sum_{k l} C_{l k} \operatorname{Tr}\left[A_{k}^{\dagger} U B_{l} \rho\right] .
$$

We can now define fidelity and coherence measures based on the maximum allowed interference for given states, in analogy to Uhlmann's fidelity for states [13] (extended to channels in Ref. [14]) $\mathcal{F}^{(\mathrm{Uhl})}\left(\rho_{A}, \rho_{B}\right)=$ $\left.\sup _{|\alpha\rangle,|\beta\rangle}\right\rangle\langle\alpha \mid \beta\rangle \mid$, where $|\alpha\rangle$ and $|\beta\rangle$ purify $\rho_{A}$ and $\rho_{B}$, respectively.

a)

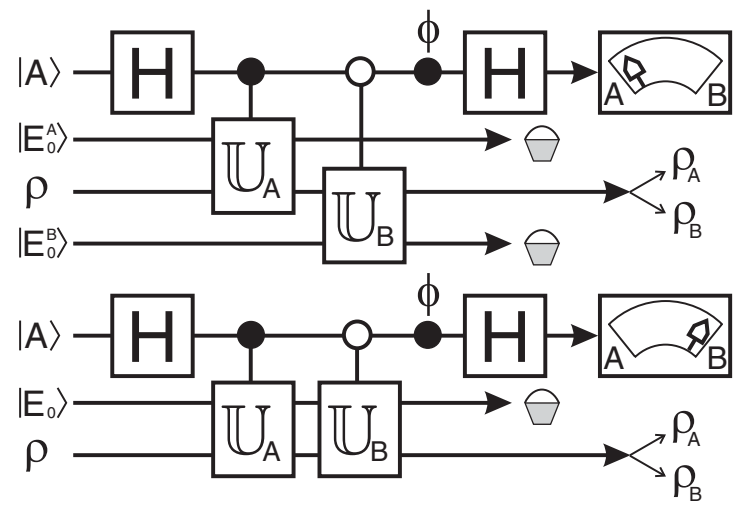

FIG. 3. (a) LSP gluing of $\Lambda_{A}$ and $\Lambda_{B}$ modeled by unitaries $\mathbb{U}_{A}$ and $\mathbb{U}_{B}$ acting on $\rho$ and separate ancillas in states $\left|E_{0}^{A}\right\rangle$ and $\left|E_{0}^{B}\right\rangle$. In an interferometric picture $\rho$ corresponds to the internal state, and $|A\rangle$ to the path state. $\mathbb{U}_{A}$ and the variable phase shift $\phi$ are conditioned upon $|A\rangle$, while $\mathbb{U}_{B}$ is conditioned upon path state $|B\rangle$. (b) SP gluing of $\Lambda_{A}$ and $\Lambda_{B}$, where the paths share a common ancilla in state $\left|E_{0}\right\rangle$. 
If the particle initially is in the internal state $|\psi\rangle$, the first beam splitter causes the superposition $|\eta\rangle=(|A\rangle|\psi\rangle+$ $|B\rangle|\psi\rangle\rangle) / \sqrt{2}$, where the orthonormal states $|A\rangle$ and $|B\rangle$ correspond to the two paths of the interferometer. We define the subspace local coherent fidelity $\mathcal{F}^{(\mathrm{LSP})}\left(\rho_{A}, \rho_{B}\right)$ as the maximal visibility achievable for all possible LSP operations preparing $\rho_{A}$ and $\rho_{B}$ in their respective path, i.e., all LSP operations $\Lambda$ such that $\langle A| \Lambda(|\eta\rangle \times$ $\langle\eta|)|A\rangle=\rho_{A} / 2$ and $\langle B|\Lambda(|\eta\rangle\langle\eta|)| B\rangle=\rho_{B} / 2$. Hence,

$$
\mathcal{F}^{(\mathrm{LSP})}\left(\rho_{A}, \rho_{B}\right)=\sup _{\|\vec{a}\|_{2},\|\vec{b}\|_{2} \leq 1}|F(|\psi\rangle\langle\psi|)|,
$$

where $\vec{a}$ and $\vec{b}$ are as in Eq. (1). We might expect that Eq. (3) would depend on the choice of marginal channels $\Lambda_{A}$ and $\Lambda_{B}$, and that we would have to optimize over all channels such that $\Lambda_{A}(|\psi\rangle\langle\psi|)=\rho_{A}$ and $\Lambda_{B}(|\psi\rangle \times$ $\langle\psi|)=\rho_{B}$. However, this is not the case as we show below, $\mathcal{F}^{(\text {LSP })}$ depends only on $\rho_{A}$ and $\rho_{B}$, and we can choose any feasible channels $\Lambda_{A}$ and $\Lambda_{B}$ to form the LSP gluings. Obviously, the choice of initial internal state $|\psi\rangle$ does not matter, as long as it is pure.

Note that $F(|\psi\rangle\langle\psi|)=\vec{a}^{\dagger} Q \vec{b}$, where $Q$ is a matrix with elements

$$
Q_{k l}=\left\langle\psi\left|A_{k}^{\dagger} B_{l}\right| \psi\right\rangle .
$$

It follows that the maximum of $|F(|\psi\rangle\langle\psi|)|$ (fixing $\Lambda_{A}$ and $\left.\Lambda_{B}\right)$ is equal to the largest singular value of $Q$.

A set of (not necessarily normalized) pure states $\left\{\left|a_{k}\right\rangle\right\}$ is a pure decomposition of $\rho_{A}$ if $\rho_{A}=\sum_{k}\left|a_{k}\right\rangle\left\langle a_{k}\right|$. Let $\left\{\left|b_{l}\right\rangle\right\}$ be a pure decomposition of $\rho_{B}$ and consider the matrix $M$ with elements $M_{k l}=\left\langle a_{k} \mid b_{l}\right\rangle$. It can be shown that the singular values of $M$ are independent of the choices of pure decompositions. In particular, we may use the spectral decompositions, with eigenvalues $\lambda_{k}^{A(B)}$ and orthonormal eigenvectors $\left|\tilde{\psi}_{k}^{A(B)}\right\rangle$, to obtain $M_{k l}=\left(\lambda_{k}^{A} \lambda_{l}^{B}\right)^{1 / 2}\left\langle\tilde{\psi}_{k}^{A} \mid \tilde{\psi}_{l}^{B}\right\rangle$. The $k$ th singular value of $M$ is $s_{k}(M)=\lambda_{k}\left(\sqrt{\sqrt{\rho_{B}} \rho_{A} \sqrt{\rho_{B}}}\right)$, where $\lambda_{k}$ is the $k$ th eigenvalue of the enclosed operator.

Returning to Eq. (4), since $\Lambda_{A}(|\psi\rangle\langle\psi|)=\rho_{A},\left\{A_{k}|\psi\rangle\right\}$ is a pure decomposition of $\rho_{A}$. Similarly, $\left\{B_{l}|\psi\rangle\right\}$ is a pure decomposition of $\rho_{B}$. Thus, the singular values of $Q$ are independent of the channels $\Lambda_{A}$ and $\Lambda_{B}$ that generate $\rho_{A}$ and $\rho_{B}$, and we obtain

$$
\mathcal{F}^{(\mathrm{LSP})}\left(\rho_{A}, \rho_{B}\right)=\lambda_{\max }\left(\sqrt{\sqrt{\rho_{B}} \rho_{A} \sqrt{\rho_{B}}}\right),
$$

where $\lambda_{\max }$ denotes the largest eigenvalue.

We define the subspace preserving coherent fidelity $\mathcal{F}^{(\mathrm{SP})}\left(\rho_{A}, \rho_{B}\right)$ similarly as for $\mathcal{F}^{(\mathrm{LSP})}$, but allowing all SP gluings. It can be shown that the maximum of $|F(|\psi\rangle\langle\psi|)|$ for all SP gluings is equal to $\sup _{C C^{\dagger} \leq I}|\operatorname{Tr}[C Q]|=$ $\sum_{k} s_{k}(Q)$, with $Q$ defined in Eq. (4). Since the singular values of $Q$ are independent of the chosen channels,

$$
\mathcal{F}^{(\mathrm{SP})}\left(\rho_{A}, \rho_{B}\right)=\operatorname{Tr} \sqrt{\sqrt{\rho_{B}} \rho_{A} \sqrt{\rho_{B}}}=\mathcal{F}^{(\mathrm{Uhl})}\left(\rho_{A}, \rho_{B}\right) .
$$

This result is also obtainable from the Stinespring con- struction of the SP gluings. For all purifications $|\alpha\rangle$ and $|\beta\rangle$ of $\rho_{A}$ and $\rho_{B}$, there are Stinespring dilations such that the resulting gluing [Fig. 3(b)] implements the transformation $(|A\rangle+|B\rangle)|\psi\rangle\left|E_{0}\right\rangle / \sqrt{2} \rightarrow(|A\rangle|\alpha\rangle+|B\rangle|\beta\rangle) / \sqrt{2}, \quad$ which has visibility $v=|\langle\alpha \mid \beta\rangle|$, for which the maximum over all purifications is the Uhlmann fidelity.

The coherent fidelities measure the coherent overlaps of the two states interfering at the beam splitter. In keeping with the notion that unitary operations preserve coherence (though not necessarily the fidelity) of states, we would like to characterize purely the coherence of a preparation. For example, if $|\psi\rangle$ and $\left|\psi^{\perp}\right\rangle$ are orthogonal, a possible global state is $\left(|A\rangle|\psi\rangle+|B\rangle\left|\psi^{\perp}\right\rangle\right) / \sqrt{2}$, but the coherent fidelity measures are zero. However, by a subspace local unitary transformation rotating $\left|\psi^{\perp}\right\rangle$ into $|\psi\rangle$, we may regain the maximal visibility reflecting this potential capacity for interference. To quantify this, we employ the generalized interferometer and define $G^{(\mathrm{LSP})}\left(\rho_{A}, \rho_{B}\right)$ between two states $\rho_{A}$ and $\rho_{B}$ as the maximal visibility that can be reached for all possible unitary shifts $U$ and for all possible LSP operations that prepare the states $\rho_{A}$ and $\rho_{B}$ [Fig. 2(b)]. If we initially fix $U$, the calculation is almost as for previous measures, except that Eq. (4) is replaced with $\tilde{Q}_{k l}=\left\langle\psi\left|A_{k}^{\dagger} U B_{l}\right| \psi\right\rangle$. We find that the singular values of this matrix are independent of the choices of feasible channels, yielding

$$
\begin{aligned}
\mathcal{G}^{(\mathrm{LSP})}\left(\rho_{A}, \rho_{B}\right) & =\sup _{U} \lambda_{\max }\left(\sqrt{\sqrt{\rho_{B}} U \rho_{A} U^{\dagger} \sqrt{\rho_{B}}}\right) \\
& =\sqrt{\lambda_{\max }\left(\rho_{A}\right)} \sqrt{\lambda_{\max }\left(\rho_{B}\right)} .
\end{aligned}
$$

Note that this measure is a product between two quantities each related only to local objects. Similarly, allowing SP operations in the generalized interferometer yields

$$
\begin{aligned}
\mathcal{G}^{(\mathrm{SP})}\left(\rho_{A}, \rho_{B}\right) & =\sup _{U} \operatorname{Tr} \sqrt{\sqrt{\rho_{B}} U \rho_{A} U^{\dagger} \sqrt{\rho_{B}}} \\
& =\sum_{k} \sqrt{\lambda_{k}^{\downarrow}\left(\rho_{A}\right)} \sqrt{\lambda_{k}^{\downarrow}\left(\rho_{B}\right)},
\end{aligned}
$$

where $\lambda_{k}^{\downarrow}$ denotes the eigenvalues of the enclosed operator sorted in a nonincreasing order.

The interferometric measures introduced in this Letter lend themselves to experiment, and can serve as tools to probe the dependence or independence of physical processes. Since these measures are independent of the marginal channels $\left(\Lambda_{A}\right.$ and $\left.\Lambda_{B}\right)$, this technique may be used even if we do not know them. If the input internal state is pure it suffices to know the states $\rho_{A}$ and $\rho_{B}$. If the visibility exceeds what is obtainable with LSP gluings, then we can conclude that the total process cannot be subspace local. More generally, the interferometric approach allows us to differentiate between processes, although these are indistinguishable if regarded as operations on the internal state of the particle. For example, consider the transverse relaxation of a qubit, turning all 
a)

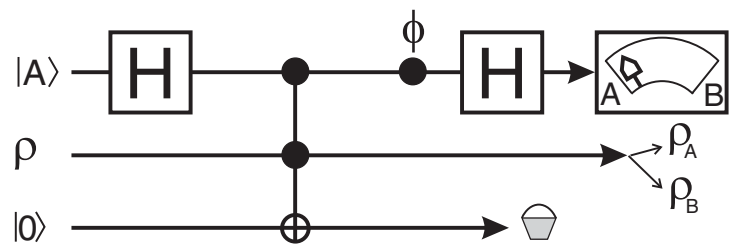

b)

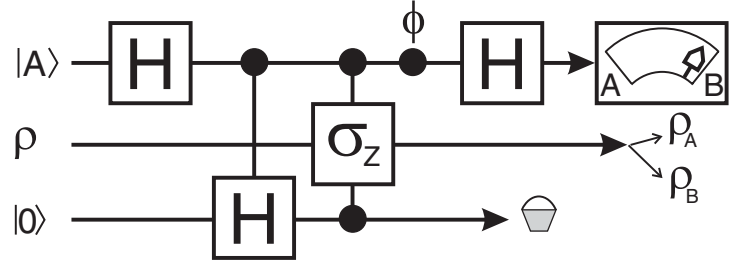

FIG. 4. In the upper path of an interferometer is a channel that converts all input states into incoherent mixtures of the basis states, i.e., transverse relaxation (or $T_{2}$ process in spin dynamics). This can arise either from, e.g., (a) an effective measurement modeled by a controlled NOT onto a measurement qubit or (b) by a Hadamard gate applied on the ancillary qubit, followed by a $\sigma_{z}$ gate conditioned on the ancilla.

input qubit states into incoherent mixtures of the $|0\rangle$ and $|1\rangle$ states. This channel can be obtained as the average output from a projective measurement of $\sigma_{Z}$ [Fig. 4(a)]. The maximum visibility obtainable with this process in one path of an interferometer is $v_{\max }=1$. The same marginal channel results from the circuit outlined in Fig. 4(b), however the maximal visibility is $v_{\max }=1 / \sqrt{2}$ in this case. Thus, the interferometer distinguishes between these two processes, although a direct process tomography on the internal state would reveal no difference.

Instead of a spatial interferometer, we can also consider temporally separated paths and processes. Time-bin photonic qubits, described in Ref. [15], propagating in a material with minimal effect on the timing of the pulses, is analogous to the path degree of freedom in Fig. 1, and photon polarization to the internal degree of freedom. If polarization decoherence is due to "classical" perturbations of the optical fiber (e.g., vibrational or thermal) the visibility could be expected to be high for short time delays, since the two pulses experience highly correlated noise and thus the gluing would be an SP operation. If the delay exceeds the autocorrelation time of the perturbations, the gluing is LSP and the visibility would drop below the threshold for independent quantum channels [1]. Determining noise correlation is important for tuning data encoding either for communication (e.g., whether to use product or entangled signal states $[16,17])$ or computation (highly correlated noise can magnify error probability for concatenated codes [18]).

In conclusion, we define interferometric measures of fidelity and coherence between states, quantifying the "quantumness" of the preparation processes in that they correspond to the capacity of the operations to preserve the ability of the particles to interfere. We define four measures based on the maximal visibility obtainable in an interfer- ometer, differing with respect to the locality or nonlocality of the preparation procedures, as well as the choice of interferometer. In the case of the standard Mach-Zender interferometer and nonlocal operations in the form of subspace preserving channels [5] we obtain the Uhlmann fidelity as the maximal visibility. The operational nature of these measures lend themselves to experiment, as well as for investigating coherence and correlation of spatially or temporally separated physical processes, which is important for the tuning of encoding and error correction schemes to the characteristics of the underlying processes causing decoherence [6]. One can consider extending the ideas presented in this Letter, allowing mixed input states, the concatenation of several channels which could be generalized to (Markovian) continuous quantum channels, and analogous measures for the channels themselves.

D. K. L. O. acknowledges the support of the CambridgeMIT Institute Quantum Information Initiative, EU grants RESQ (No. IST-2001-37559) and TOPQIP (No. IST-200139215), EPSRC QIP IRC (UK), and Sidney Sussex College, Cambridge. J. A. acknowledges support from the Swedish Research Council.

*Electronic address: J.Aberg@damtp.cam.ac.uk

[1] D. K. L. Oi, Phys. Rev. Lett. 91, 067902 (2003).

[2] J. Åberg, Phys. Rev. A 70, 012103 (2004).

[3] B.-G. Englert, Phys. Rev. Lett. 77, 2154 (1996).

[4] S. Pancharatnam, Proc. Indian Acad. Sci., Sect. A 44, 247 (1956).

[5] J. Åberg, Ann. Phys. (N.Y.) 313, 326 (2004).

[6] P. P. Rohde, G. J. Pryde, J.L. O'Brien, and T. C. Ralph, Phys. Rev. A 72, 032306 (2005).

[7] We cannot directly interpret this as resulting from the distinguishability of the interfering states, but by considering purifications of the mixed states, we recover the interpretation.

[8] E. Sjöqvist, A. K. Pati, A. Ekert, J.S. Anandan, M. Ericsson, D. K. L. Oi, and V. Vedral, Phys. Rev. Lett. 85, 2845 (2000).

[9] M. Ericsson, E. Sjöqvist, J. Brännlund, D. K. L. Oi, and A. K. Pati, Phys. Rev. A 67, 020101(R) (2003).

[10] J. G. Peixoto de Faria, A. F. R. de Toledo Piza, and M. C. Nemes, Europhys. Lett. 62, 782 (2003).

[11] K. Kraus, States, Effects, and Operations (SpringerVerlag, Berlin, 1983).

[12] W. F. Stinespring, Proc. Am. Math. Soc. 6, 211 (1955).

[13] A. Uhlmann, Rep. Math. Phys. 9, 273 (1976).

[14] M. Raginsky, Phys. Lett. A 290, 11 (2001).

[15] J. Brendel, N. Gisin, W. Tittel, and H. Zbinden, Phys. Rev. Lett. 82, 2594 (1999).

[16] C. Macchiavello, G. M. Palma, and S. Virmani, Phys. Rev. A 69, 010303(R) (2004).

[17] N. J. Cerf, J. Clavareau, C. Macchiavello, and J. Roland, Phys. Rev. A 72, 042330 (2005).

[18] J.P. Clemens, S. Siddiqui, and J. Gea-Banacloche, Phys. Rev. A 69, 062313 (2004). 